\title{
PFLEIDERER 2: IDENTIFICATION OF A NEW GLOBULAR CLUSTER IN THE GALAXY*
}

\author{
S. Ortolani ${ }^{1}$, C. Bonatto ${ }^{2}$, E. Bica ${ }^{2}$, and B. Barbuy ${ }^{3}$ \\ ${ }^{1}$ Università di Padova, Dipartimento di Astronomia, Vicolo dell'Osservatorio 5, I-35122 Padova, Italy; sergio.ortolani@ unipd.it \\ ${ }^{2}$ Universidade Federal do Rio Grande do Sul, Departamento de Astronomia, CP 15051, Porto Alegre 91501-970, Brazil; charles@if.ufrgs.br, bica@if.ufrgs.br \\ ${ }^{3}$ Universidade de São Paulo, Departamento de Astronomia, Rua do Matão 1226, São Paulo 05508-900, Brazil; barbuy @ astro.iag.usp.br \\ Received 2009 February 21; accepted 2009 July 3; published 2009 August 5
}

\begin{abstract}
We provide evidence that indicates the star cluster Pfleiderer 2, which is projected in a rich field, as a newly identified Galactic globular cluster. Since it is located in a crowded field, core extraction and decontamination tools were applied to reveal the cluster sequences in $B, V$, and $I$ color-magnitude diagrams (CMDs). The main CMD features of Pfleiderer 2 are a tilted red giant branch and a red horizontal branch, indicating a high metallicity around solar. The reddening is $E(B-V)=1.01$. The globular cluster is located at a distance of $d_{\odot}=16 \pm 2 \mathrm{kpc}$ from the Sun. The cluster is located $2.7 \mathrm{kpc}$ above the Galactic plane and at a distance of $R_{\mathrm{GC}}=9.7 \mathrm{kpc}$ from the Galactic center, which is unusual for a metal-rich globular cluster.
\end{abstract}

Key words: globular clusters: individual (Pfleiderer 2) - Hertzsprung-Russell (HR) diagram

Online-only material: color figures

\section{INTRODUCTION}

Classical globular clusters are prominent objects with respect to the surrounding fields. In recent years, however, lowluminosity globular clusters have been identified or discovered in the Galaxy. These include obscured or field-contaminated objects, or a combination of these factors (e.g., Ortolani et al. 2006, and references therein). Kurtev et al. (2008) recently found GLIMPSE-C02, an extremely obscured and metal-rich globular cluster, which was almost simultaneously detected by Strader $\&$ Kobulnicky (2008).

Pfleiderer 2 was found by Pfleiderer et al. (1977) and has also been referred to as PWM2. The cluster center is located at $(\mathrm{J} 2000) \alpha=17^{\mathrm{h}} 58^{\mathrm{m}} 40^{\mathrm{s}}, \delta=-5^{\circ} 04^{\prime} 30^{\prime \prime}$, with Galactic coordinates $\ell=22.28, b=+9.32$. The angular size is $\sim 2.5$, as seen on DSS/XDSS ${ }^{4}$ images.

In Section 2, the observations and reductions are described. In Section 3, we describe the decontamination procedures. Colormagnitude diagrams (CMDs) are presented and fundamental parameters are derived in Section 4. The cluster structure is studied in Section 5. Concluding remarks are given in Section 6.

\section{OBSERVATIONS}

Pfleiderer 2 was observed on 2008 June 5, 6, with the $3.58 \mathrm{~m}$ Galileo telescope (TNG) at La Palma, equipped with the Dolores spectrograph focal reducer. An EEV 4260 CCD detector with $2048 \times 2048$ pixels, of pixel size $13 \mu \mathrm{m}$, was used. A pixel corresponds to 0 '.252 on the sky, and the full field of the camera is $8.6 \times 8.6$. The $\log$ of observations is provided in Table 1 . Figure 1 shows a $420 \mathrm{~s}$ full field $I$ image of Pfleiderer 2, showing a rather prominent cluster.

\subsection{Pfleiderer 2 Calibrations}

The calibration was performed using three different Landolt (1983, 1992) standard fields observed in BVI, the first night

\footnotetext{
* Based on observations made with the Italian Telescopio Nazionale Galileo (TNG) operated on the island of La Palma, by the Fundación Galileo Galilei of INAF (Istituto Nazionale di Astrofisica) at the Spanish Observatorio del Roque de los Muchachos of the Instituto de Astrofisica de Canarias.

4 Extracted from the Canadian Astronomy Data Centre (CADC)—http://cadcwww.dao.nrc.ca/.
}

(PG1633+099, SA 113337, PG 2213-06) with different exposure times and in different positions. We checked shutter time dependence and zero-point variations across the field. On the second night (June 6), five additional Landolt (1992) fields were observed (PG 1323-086, PG1528+062, PG1633+099, SA113 $337, \mathrm{PG} 2213-06)$. The airmass corrections of 0.15 in $V, 0.25$ in $B$, and 0.07 in $I$ have been done using the standard values given for La Palma on clear nights (with no dust in the atmosphere), available at the DOLORES Web site. ${ }^{5}$

The derived calibration equations are

$$
\begin{aligned}
V & =28.44-0.06(V-I)+v, \\
I & =28.28-0.07(V-I)+i \\
B & =28.59+0.12(B-V)+b, \\
V & =28.43-0.06(B-V)+v,
\end{aligned}
$$

where the zero points are calculated for $10 \mathrm{~s}$ exposures at 1.15 airmasses, with an aperture of $10^{\prime \prime} \times 1^{\prime \prime}$. The bvi are instrumental magnitudes (counts in ADUs, gain 1e-/ADU). The $B V I$ are the calibrated magnitudes. The zero points were remarkably stable and identical on the two nights. No shutter time effects have been detected at the $0.01 \mathrm{mag}$ level in the exposure range of our images (from 1 to $30 \mathrm{~s}$ ). In the $1000 \times 1000$ pixels central area the standard stars showed a very good uniformity, but their flux rapidly decreased at the frame corners. The repeated measurements of the standard stars across the field have been used to separate the contribution of the sky concentration from the flat field, including the decrease of sensitivity of the system (possibly due to some vignetting) at the edge. Measurements performed with different apertures excluded the possibility that the loss of light at the edges was due to the degradation of the point-spread function (PSF).

\subsection{Reductions}

The CCD frame reductions were performed using a slightly more complex procedure than the standard flat field division. This was needed due to the analysis of the standard star fluxes across the field, compared to the background brightness, and showed a sky flux excess in an area roughly corresponding

\footnotetext{
5 http://www.tng.iac.es/info/la_palma_sky.html
} 
Table 1

Log of Observations

\begin{tabular}{lcrcc}
\hline \hline Filter & Date & Exp $(\mathrm{s})$ & Seeing $\left({ }^{\prime \prime}\right)$ & Airmass \\
\hline$V$ & 2008 Jun 5-6 & 600 & 1.1 & 1.2 \\
$I$ & “ & 30 & 1.2 & 1.2 \\
$I$ & “ & 420 & 1.1 & 1.2 \\
$V$ & 2008 Jun 6-7 & 30 & 1.6 & 1.7 \\
$V$ & “ & 900 & 1.6 & 1.6 \\
$B$ & “ & 60 & 1.5 & 1.5 \\
$B$ & “ & 20 & 1.4 & 1.5 \\
$B$ & “ & 900 & 1.4 & 1.4 \\
$B$ & “ & 60 & 1.4 & 1.4
\end{tabular}

to the central 500 pixels. This excess is interpreted as the effect called "sky concentration" (Andersen et al. 1995). ${ }^{6}$ This is known to be caused by internal reflections and is color dependent. In our images it reached about $6 \%$ in $I, 5 \%$ in $V$ (Figure 2), and was almost negligible in $B$. If the light concentration is not removed from the flat fields, it causes a systematic photometric error across the field of the same amount. The light loss at the edges was instead almost constant with the colors, up to 5\% from center to corner. The flat fielding (after bias correction) was performed in four main steps: (1) a map of sky concentration was prepared using the standard stars, compared to the sky level; (2) the contribution of the light concentration was extracted from the flat fields and smoothed (Figure 2); (3) the light concentration was scaled to the value of the sky images and subtracted; (4) the flat field obtained after removing the light concentration was used to correct the high frequencies and sensitivity variations (including vignetting).

The corrected images have been processed using Daophot II and Allstars (Stetson 1987, 1994). The software is available in MIDAS for the instrumental photometry of individual stars, and was converted into the standard Johnson-Cousins system, defined from the Landolt stars, using the above calibration equations. The conversion from the instrumental (PSF-

\footnotetext{
6 http://www.ls.eso.org/lasilla/Telescopes/2p2T.old.obsolete/D1p5M/
} RepsFinal/Final/gain_calibration.ps

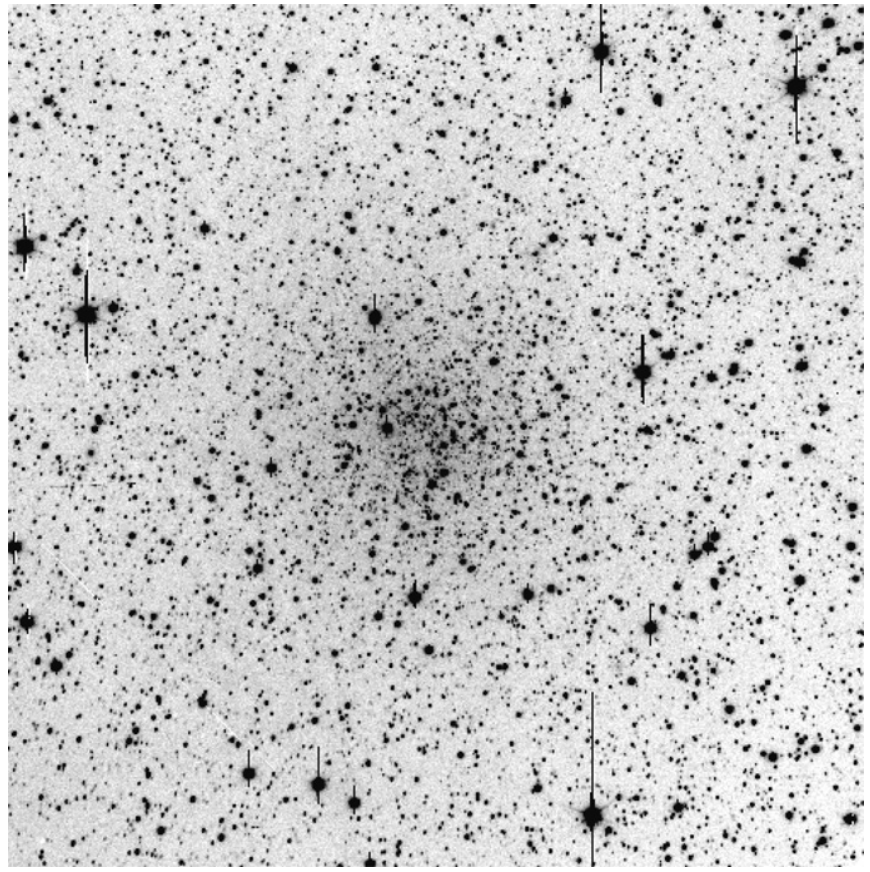

Figure 1. Pfleiderer 2 full field $420 \mathrm{~s} I$ image. East is to the right and north is to the top.

convolved) magnitudes to the calibrated ones revealed an additional problem related to the image-quality variation across the field. This produces a variable aperture correction, changing with time and filter. In Pfleiderer 2 images, it was of the order of $0.02 \mathrm{mag}$, with a peak around $0.04 \mathrm{mag}$ at the extreme corners. For this reason, we adopted the aperture correction established from stars located in the central 200 pixels which is representative of most of the image within about 1\%-2\%.

The resulting calibration accuracy, taking into account the uncertainties in the calibration equations $(\sim 0.01 \mathrm{mag})$, the flatfield procedure (an additional 0.01-0.02 mag), and the aperture corrections $(0.02 \mathrm{mag})$, is estimated to be within \pm 0.04 in a single filter. The relative photometric errors have been derived
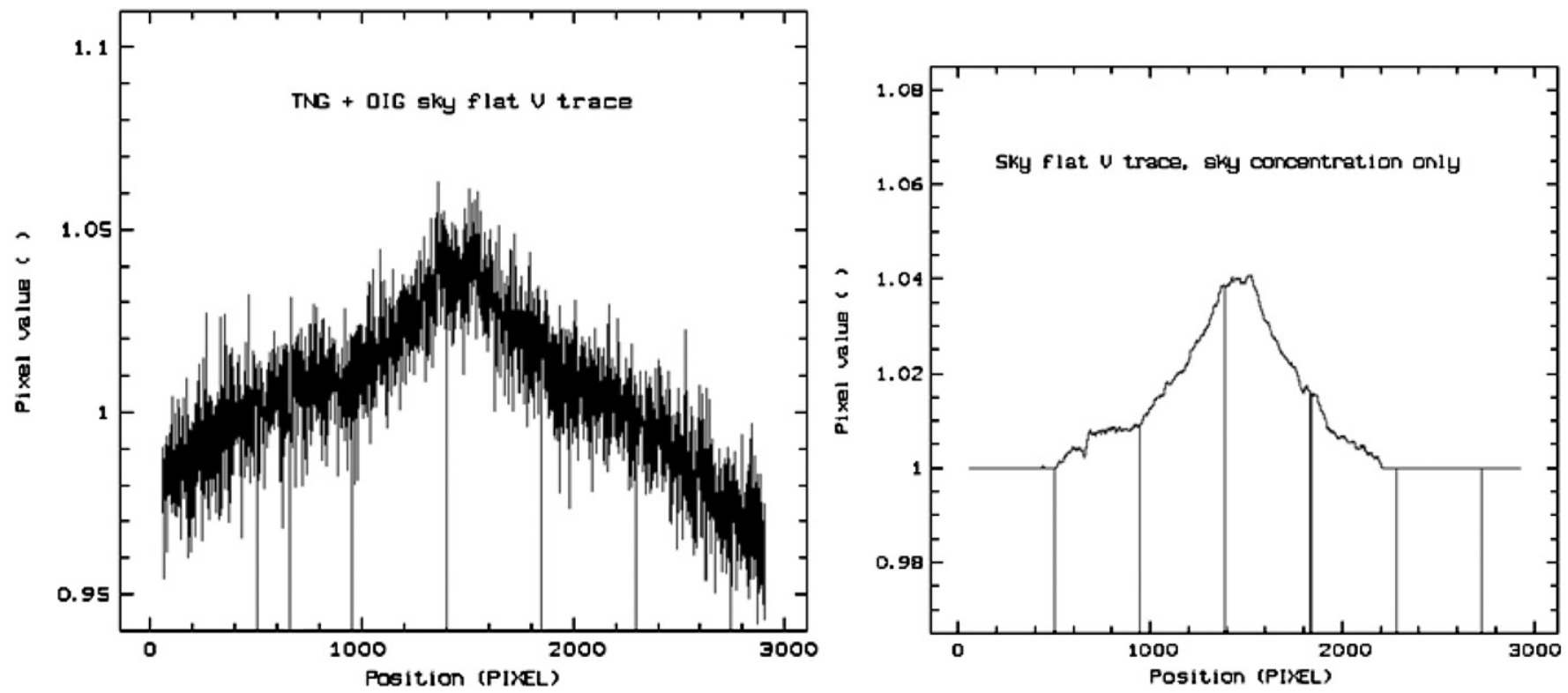

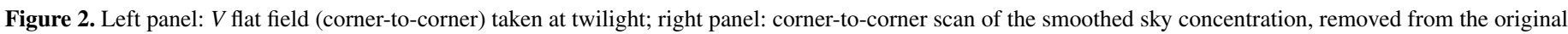
flat field. 

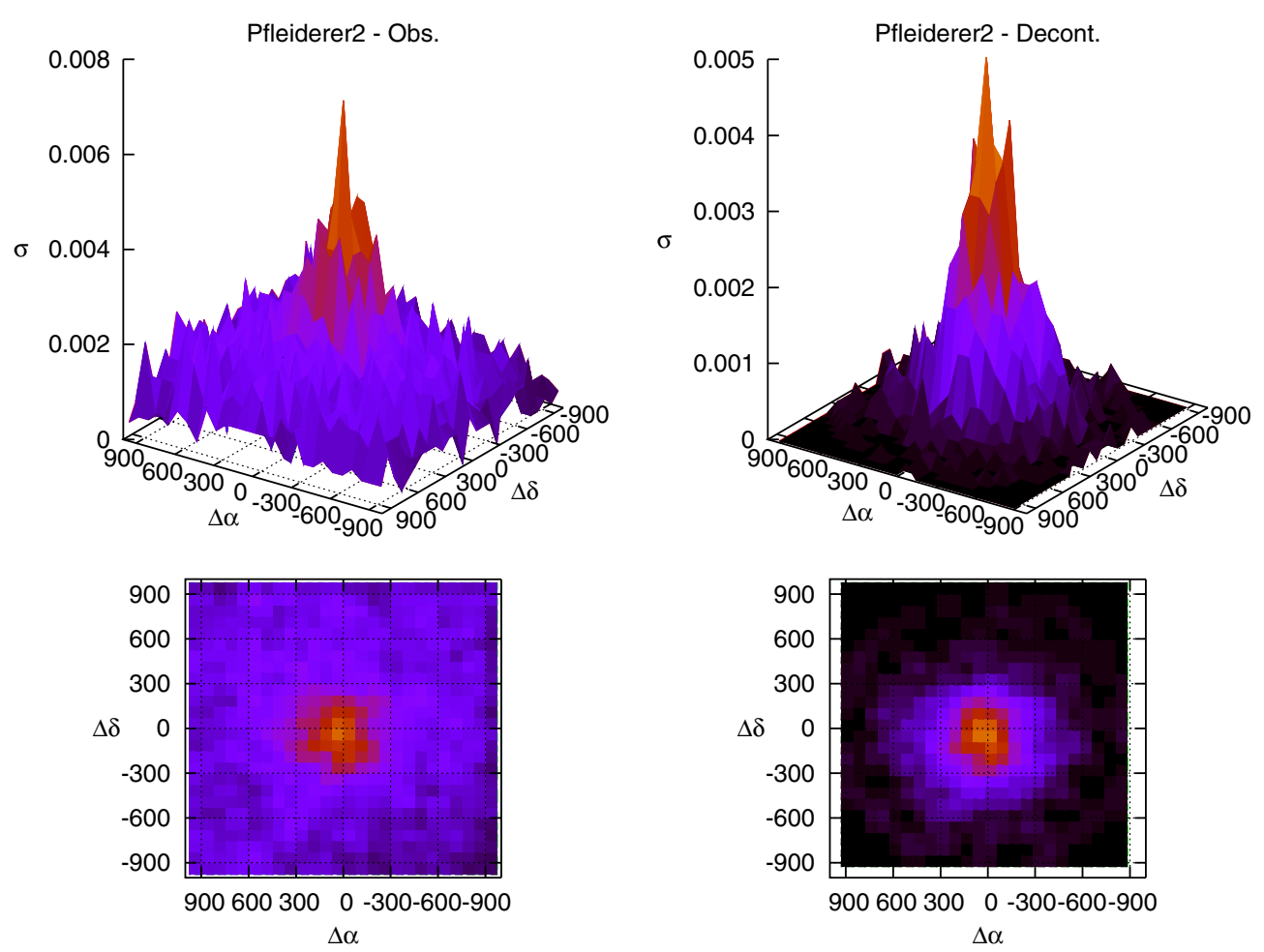

Figure 3. Pfleiderer 2: surface density distribution built with the raw (left panels) and decontaminated photometry (right panels). $\sigma$ is given in units of stars pixel ${ }^{-2}$; $\Delta \alpha$ and $\Delta \delta$ are in pixel.

(A color version of this figure is available in the online journal.)

from short exposure (15 s in $V$ and $10 \mathrm{~s}$ in $I$ ) frame-to-frame images. The errors are $0.03 \mathrm{mag}$ for $14<V<16.5$, increasing to about $0.2 \mathrm{mag}$ at $V=20.5$. In order to have the errors for our deep $600 \mathrm{~s}$ exposures, they must be scaled to the corresponding magnitudes. The $600 \mathrm{~s}$ images imply a factor 40 larger in exposure times, and the corresponding error values can be estimated from the ones given above.

\section{DECONTAMINATION PROCEDURES}

Field stars are an important contaminant of CMDs in rich fields, especially near the disk and bulge. The decontamination algorithm employed here is based on a three-dimensional routine designed for the wide-field photometry of Two Micron All Sky Survey data, as developed in Bonatto \& Bica (2007), Bica et al. (2008), and Bica \& Bonatto (2008). In the present case, we adapted the original algorithm to work with photometry obtained with a large telescope and a single color. For clarity, we recall the basic procedures. The algorithm divides the whole magnitude and color ranges into a grid of CMD cells. For a given cluster extraction and comparison field, it estimates the relative number densities of probable field and cluster stars present in each cell. The estimated number of field stars is subsequently subtracted from each cell. Decontamination results for different cluster/field contrasts are given and discussed in the above-cited papers. The reference cell dimensions are $\Delta \mathrm{mag}=1.0$ and $\Delta$ color $=0.2$. However, to minimize spurious results, several runs of the decontamination procedure are used, with different input parameters.

In this paper, different cell sizes are considered, with $\Delta$ mag and $\Delta$ color taken from $0.5,1.0$, and 2.0 times the reference values. Also, the cell grid is shifted by $-1 / 3,0$, and $+1 / 3$ of

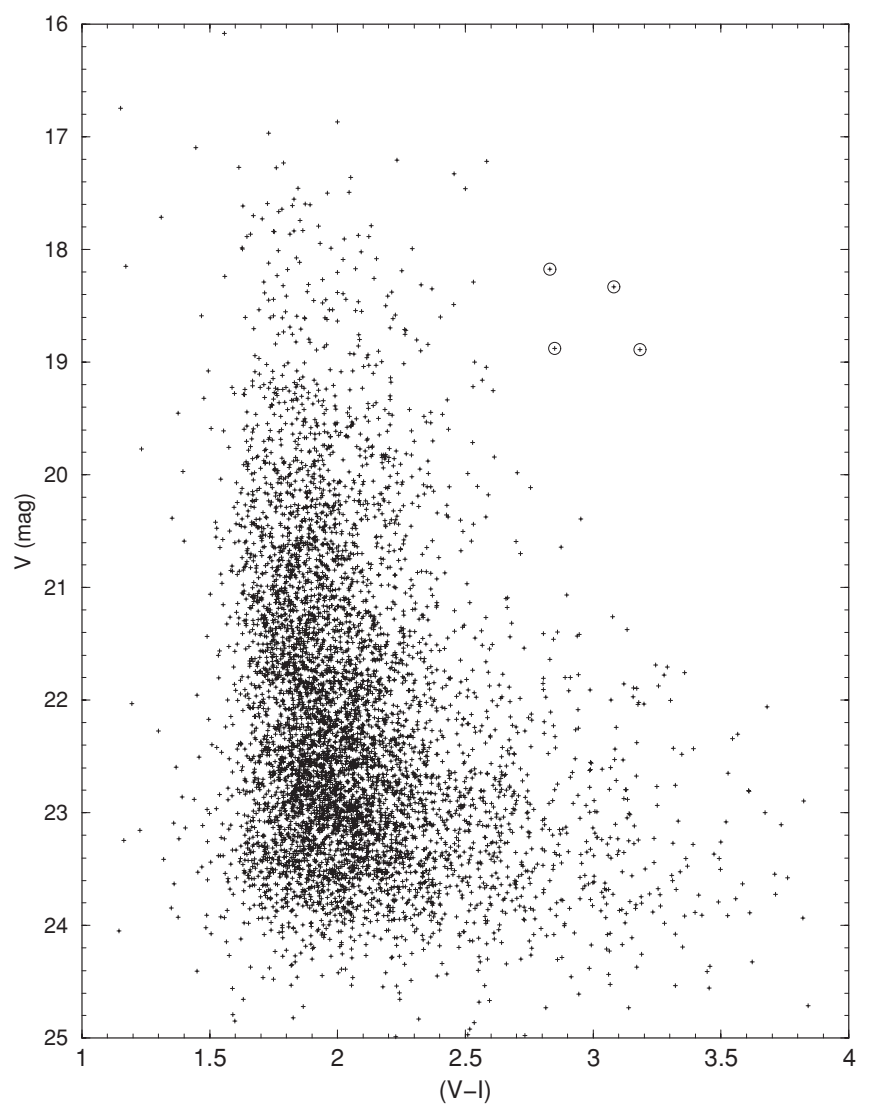

Figure 4. $V$ vs. $V-I$ CMD of Pfleiderer 2 for an extraction of $r<1000$ pixels or $r<4.2$ (essentially the full field). The bright and red stars (circled) suggest a metal-rich cluster. 

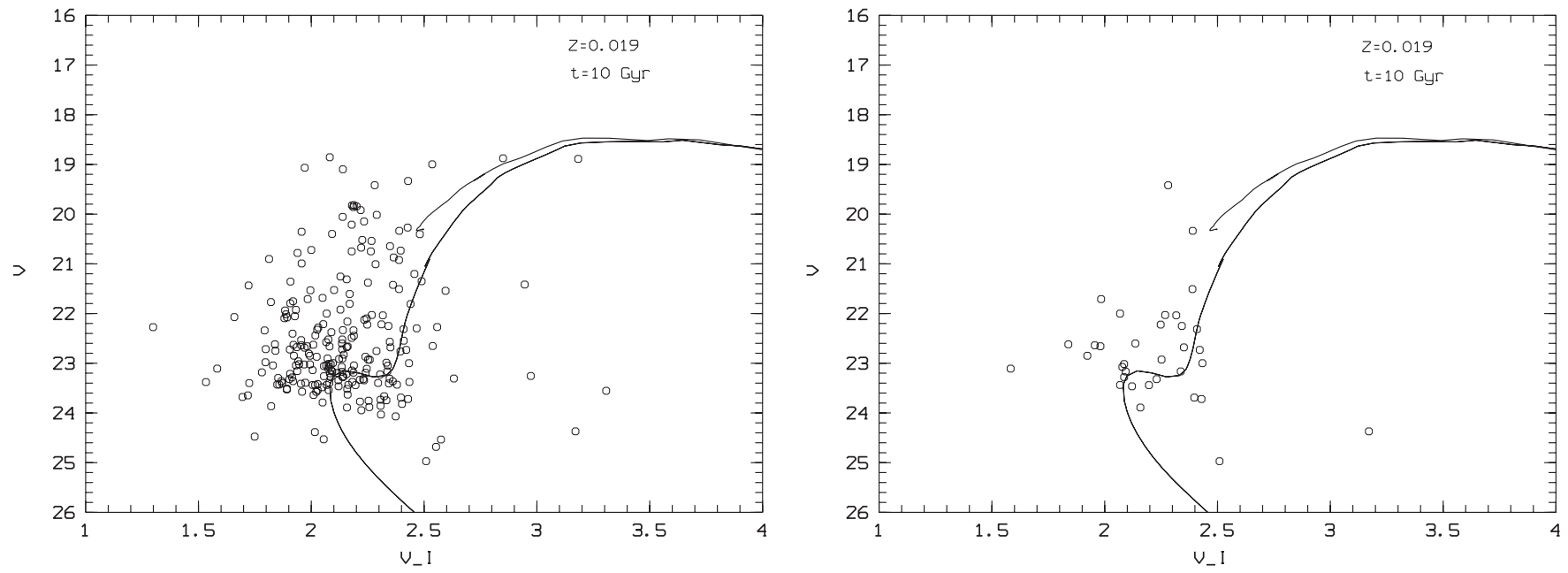

Figure 5. Solar metallicity Padova isochrone of $10 \mathrm{Gyr}$ fit to the $V$ vs. $V-I$ CMD of Pfleiderer 2 for extractions of $30^{\prime \prime}$ (left) and $10^{\prime \prime}$ (right),

the respective cell size in both the color and magnitude axes. Taking together all the grid/cell size setups, we are left with 81 different and independent decontamination combinations. Stars are ranked according to the number of times they survive each run. Finally, only the highest ranked stars are considered as cluster members and transposed to the respective decontaminated CMD. In the case of Pfleiderer 2, the decontaminated stars have a survival frequency above $S_{d}=70 \%$. We note that the two bright and red stars (Figures 6 and 7) are among those with $S_{d}=100 \%$, which implies that the observed field is scarcely populated of stars with similar color and magnitude.

In Figure 3, we investigate the surface density ( $\sigma$, in units of stars pixel ${ }^{-2}$ ) distribution of Pfleiderer 2, both with the observed (left panels) and decontaminated (right panels) photometry. We also include the respective isopleth surface maps (bottom panels), which show a rather circular geometry in the stellar distribution around the center of Pfleiderer 2. As expected, the cluster is much more contrasted in the decontaminated data.

\section{COLOR-MAGNITUDE DIAGRAMS}

Figure 4 shows the $V$ versus $V-I C M D$ of essentially the full field $(r<1000$ pixels, or $r<4$.2) around Pfleiderer 2, reaching the photometric limit of $V \approx 24$. As anticipated in Section 3, field contamination is dominant in this direction. A few bright and red stars show up at $V \approx 18.5$ and $(V-I) \approx 3$, which might suggest a metal-rich cluster.

The fit of Padova isochrones (Girardi et al. 2000) to the $V$ versus $V-I$ CMD of Pfleiderer 2 is shown in Figure 5 for extractions of $r<10^{\prime \prime}$ and 30". A tilted red giant branch (RGB) is seen, together with a clump of stars at $V=20.37 \pm 0.05$ and $(V-I)=2.4 \pm 0.1$, compatible with a red horizontal branch (HB). A tilted RGB is indicative of a high metallicity as discussed in Ortolani et al. (1991). We also note that the spread across the diagram is due to differential reddening.

The fit of the isochrones to the cluster sequences in these figures required a high metallicity of $Z=0.019([\mathrm{Fe} / \mathrm{H}] \approx 0.0)$. It might be one of the most metal-rich globular clusters in the Galaxy. The age estimated is $10 \pm 2 \mathrm{Gyr}$, compatible with a globular cluster.

This leads to a reddening of $E(V-I)=1.34$. Assuming $E(V-I) / E(B-V)=1.33$ (Dean et al. 1978), we get $E(B-V)=1.01 \pm 0.15$, which corresponds to $A_{\mathrm{V}}=3.13$. This reddening value is compatible with the dust emission maps
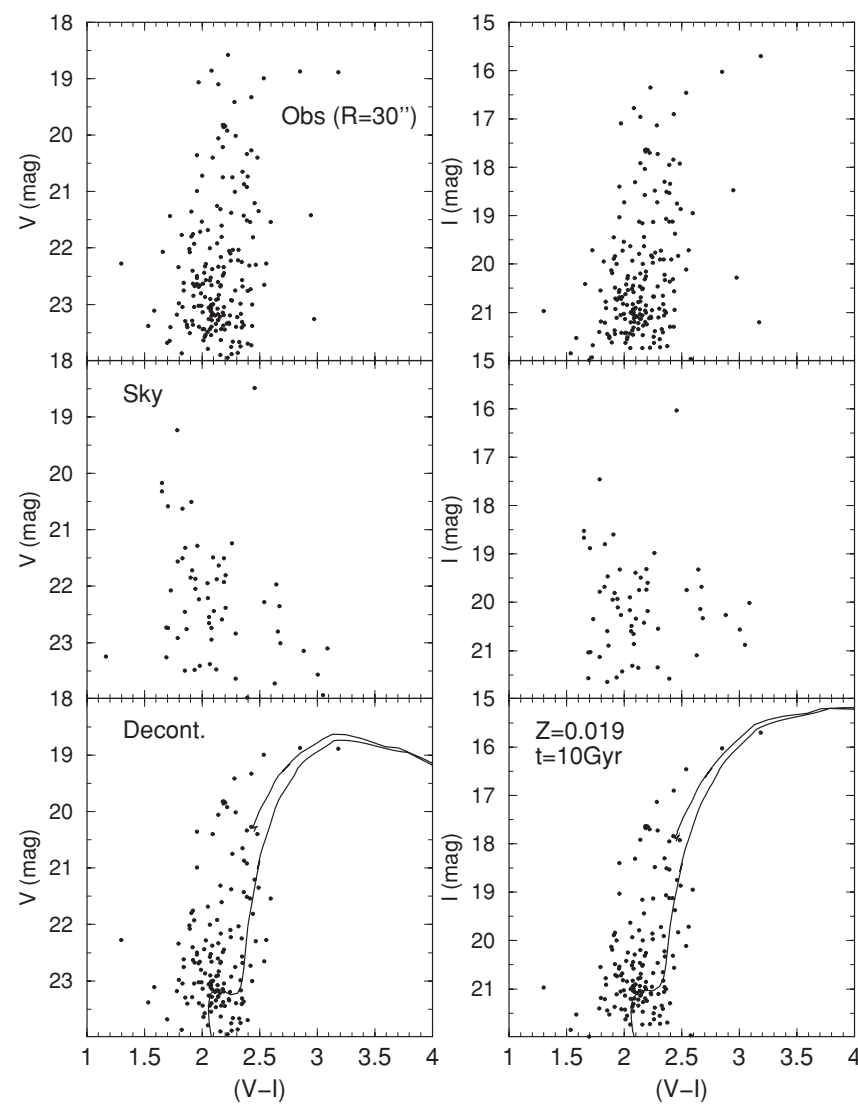

Figure 6. $V$ vs. $V-I$ and $I$ vs. $V-I$ CMD extraction of Pfleiderer 2 for $r<120$ pixels $\left(r<30^{\prime \prime}\right)$. Upper panels: observed; middle panels: equalarea sky extractions; lower panels: decontaminated CMDs with the isochrone solution from Figure 5.

by Schlegel et al. (1998), of $E(B-V)=1.15$. We derive an observed distance modulus of $(m-M)_{\mathrm{V}}=19.2$, an absolute distance modulus of $(m-M)_{\circ}=16.07$, and the distance from the Sun of $d_{\odot}=16.4 \pm 2 \mathrm{kpc}$.

Assuming the conservative distance of the Sun to the Galaxy center to be $R_{\odot}=8.0 \mathrm{kpc}$ (Reid 1993), the Galactocentric coordinates are $X=7.0 \mathrm{kpc}(X>0$ is on the other side of the Galaxy), $Y=6.1 \mathrm{kpc}$, and $Z=2.7 \mathrm{kpc}$, and the distance to the Galactic center is $R_{\mathrm{GC}}=9.7 \pm 2 \mathrm{kpc}$. 

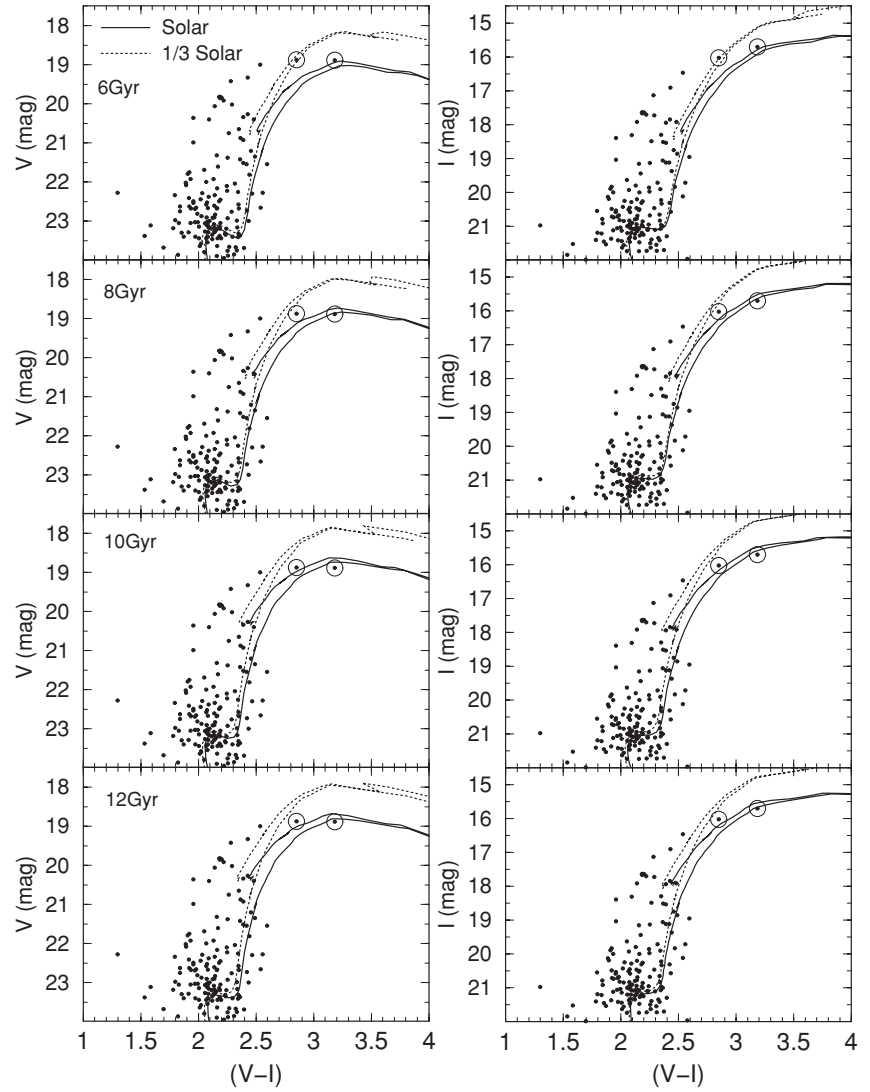

Figure 7. Alternative age and metallicity solutions applied to the $r<30^{\prime \prime}$ decontaminated CMD. Acceptable fits are obtained with nearly solar metallicities and ages within $8-10$ Gyr. The bright and red stars that support the metal-rich nature of this cluster are highlighted by circles.

A metal-rich globular cluster located far from the center is not a unique case. Terzan 7 with a 47 Tuc-like metallicity, in the Sagittarius dwarf, is even farther from the Galactic center at $R_{\mathrm{GC}}=15.9 \mathrm{kpc}$ (Salaris \& Weiss 2002). However, Pfleiderer 2 is apparently not related to any dwarf galaxy and is more metal-rich. The metal-rich cluster Palomar 8 has $[\mathrm{Fe} / \mathrm{H}]=-0.48$ and $R_{\mathrm{GC}}=5.6 \mathrm{kpc}$ (Harris 1996, hereafter $\mathrm{H} 96)$, whereas Palomar 11 has $[\mathrm{Fe} / \mathrm{H}]=-0.39$ (H96) and $R_{\mathrm{GC}}=9 \mathrm{kpc}$ (Lewis et al. 2006).

Therefore, Pfleiderer 2 has at least two other analog metalrich globular clusters located far from the bulge. In case there is no relation to a dwarf galaxy, it might be that these clusters are at apogalacticon, possibly tracing such limit for the bulge.

\subsection{Decontaminated TNG Photometry}

We applied the decontamination tools to the TNG photometry, as described in Section 3. A mosaic of CMDs extracted within 120 pixels $\left(r<30^{\prime \prime}\right)$ is given in Figure 6. The upper panels show the observed $V$ versus $V-I$ and $I$ versus $V-I$ CMDs, while the respective equal-area sky extraction is given in the middle panels. The lower panels show the statistically decontaminated CMDs, derived according to Section 3 using as comparison field the wide ring located within $750<r$ (pixel) $<950$ $\left(3.13<r\left(^{\prime}\right)<3.96\right)$.

The RGB and red HB, and a populous turnoff are seen, features that are clearer in the decontaminated plot. These features are also seen in the $V$ versus $B-V$ subtracted diagram, although not as deep as in $V$ versus $V-I$ (Figure 6). At this point, it is important to recall that the two bright and red stars

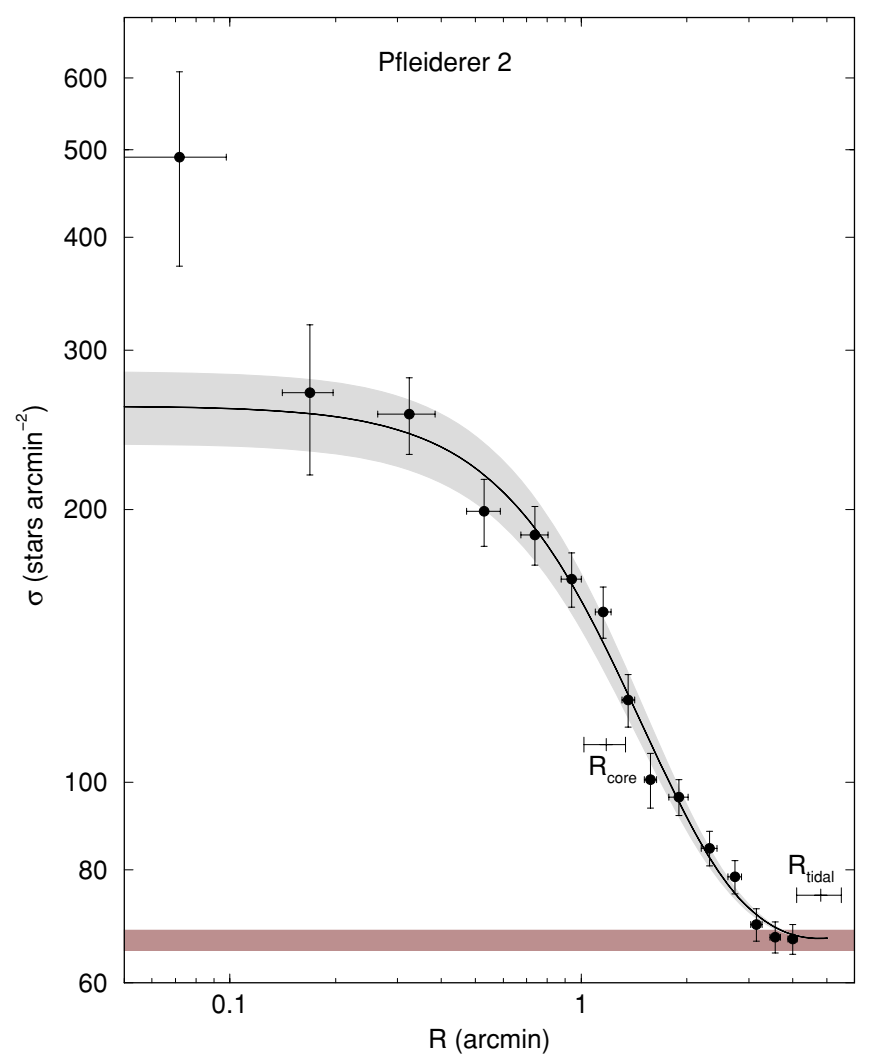

Figure 8. Pfleiderer 2 stellar radial density profile. The solid line corresponds to the best-fit three-parameter King-like profile. The shaded area corresponds to the $1 \sigma$ King fit uncertainty. Horizontal shaded bar is the background level. The cluster core and tidal radii are indicated.

(A color version of this figure is available in the online journal.)

$((V-I) \approx 3, V \approx 19)$ have a survival frequency of $100 \%$, with respect to the decontamination procedure (Section 3). Besides, the redder one is located at about $14^{\prime \prime}$ from the cluster center, while the other at about 29". Taken together, these arguments imply a high probability of both being cluster members, and suggest a metal-rich cluster.

The CMD sequences seen in these decontaminated diagrams are similar to those obtained from central extractions (Figure 5). As a caveat we note that the above isochrone solutions place the turnoff at $V \approx 23$ and $I \approx 21$, which corresponds to about 1 mag brighter than the photometric limit, and probably close to the completeness limit. However, the comparison with the field CMD (Figure 6) shows that the excess of stars at $V \gtrsim 22.5$ is real.

Finally, in Figure 7, we test alternative isochrone solutions that explore a range of ages and metallicities. We test isochrones of $6,8,10$, and $12 \mathrm{Gyr}$, both of solar and $1 / 3$ solar metallicity. Assuming that the two bright and red stars are members, the metallicity turns out clearly constrained to nearly solar, since lower metallicities would require brighter GB stars. The 6 Gyr isochrones, although producing a reasonable fit of the turnoff and RGB, fail to describe both bright and red stars simultaneously. Although we adopted $10 \mathrm{Gyr}$ as the best age solution, a spread of \pm 2 Gyr is acceptable. In the case of $8 \mathrm{Gyr}$, Pfleiderer 2 might be one of those young globular clusters such as Palomar 1 (Rosenberg et al. 1998).

\section{STRUCTURE}

In Figure 8, we give the cluster stellar radial density distribution (RDP). The central parts of the cluster show evidence 

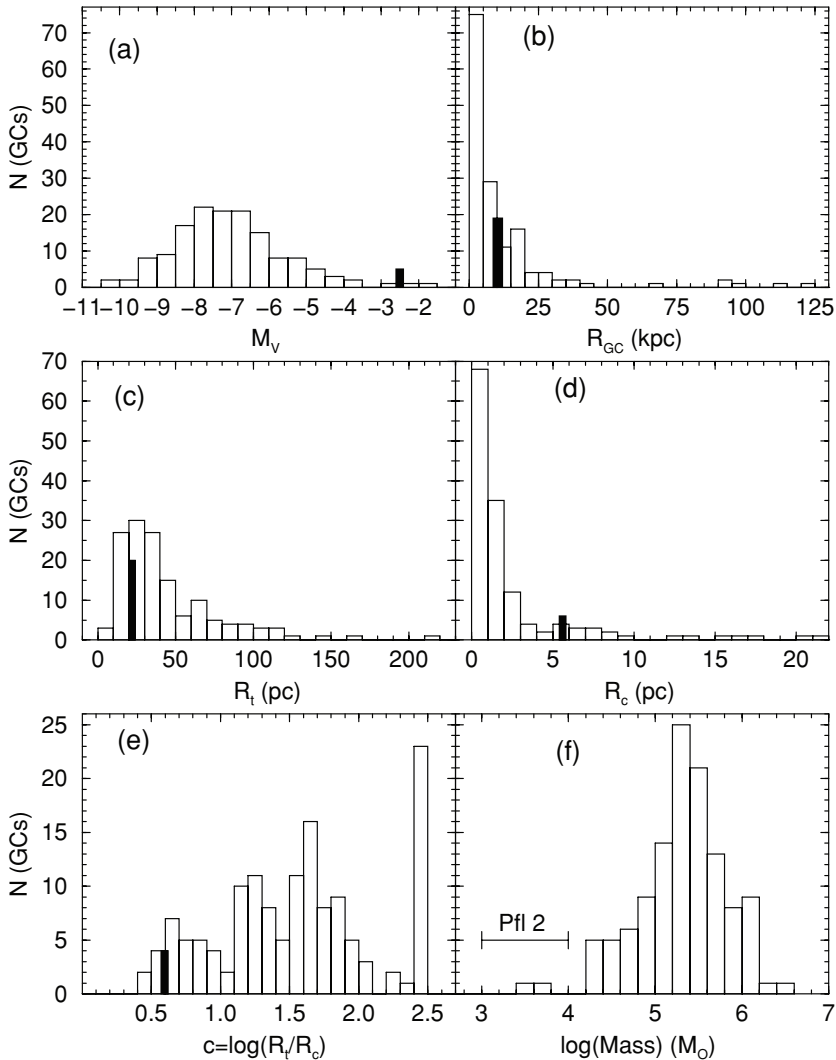

Figure 9. Astrophysical parameters of Pfleiderer 2 (black dash) are compared to those of the Galactic globular clusters (histograms). Panels show the absolute $V$ magnitude (a), Galactocentric distance (b), King tidal (c) and core (d) radii, concentration parameter (e), and mass (f). Histograms are built with $\mathrm{H} 03$ data, except the mass (f), taken from Gnedin \& Ostriker (1997).

of a post-core collapse, as seen in a number of other globular clusters, especially in the bulge (Trager et al. 1995). Otherwise, a smooth fit with the three-parameter King profile ${ }^{7}$ is obtained throughout the rest of the cluster. This tentative fit provides a core radius of $r_{\mathrm{c}}\left({ }^{\prime}\right)=1.18 \pm 0.16(\approx 5.6 \mathrm{pc})$ and a tidal radius of $r_{\mathrm{t}}\left({ }^{\prime}\right)=4.8 \pm 0.7(\approx 22 \mathrm{pc})$.

Innanen et al. (1983) determined tidal radii of globular clusters as a function of the estimated perigalacticon distances. Pfleiderer 2 compared to their sample appears to be among the intrinsically smallest globulars, only comparable with the central bulge clusters NGC 6522 and NGC 6528. In case the tidal radius is essentially determined by the perigalacticon tidal forces, the small size of this cluster suggests that it may reach the outer bulge (see Equation (4) in Innanen et al. 1983).

On the other hand, the total absolute magnitude, obtained by adding the stellar fluxes for the stars brighter than $V=24$ present in the decontaminated CMD extracted within 700 pixels, amounts to $M_{\mathrm{V}}=-2.5$. This value would place Pfleiderer 2 among the least luminous globular clusters in the Galaxy, like Palomar clusters. Therefore, if its mass is as low as that of Palomar clusters such as Palomar 12 and Palomar 13 (Gratton \& Ortolani 1988; Ortolani et al. 1985), estimated to be of $10^{3}-10^{4} M_{\odot}$, then a perigalacticon distance of $5-10 \mathrm{kpc}$ is inferred. In this case, it might be a low-mass globular cluster that has been losing mass, or be an old open cluster, with a nearly circular orbit.

\footnotetext{
7 Applied to star counts, this function is similar to that introduced by King (1962) to describe the surface brightness profiles of globular clusters.
}

To put Pfleiderer 2 into perspective, in Figure 9, we compare some of the astrophysical parameters derived in this paper with those of the globular clusters in H96 (updated in 2003; H03), together with the mass (panel d) taken from Gnedin \& Ostriker (1997). As anticipated above, Pfleiderer 2 is located in the low-luminosity tail of the globular cluster distribution (panel a) and not far from the Galactic center (panel b). The King tidal radius (panel c) is relatively small, consistent with its location in the Galaxy, while the King core (panel d), which encompasses the collapsed core (Figure 8), is clearly an upper limit. Disregarding the collapsed core, its King concentration parameter would suggest a relatively loose cluster (panel e). Undoubtedly, Pfleiderer 2 is a low-mass cluster (panel f).

\section{CONCLUDING REMARKS}

The present observations deal with a faint star cluster that we identified as a globular cluster. Previously, Pfleiderer 2 was classified as an open cluster. It is obscured in the optical, with $A_{V}=3.1$, and affected by differential reddening. Deep photometry was collected with the $3.58 \mathrm{~m} \mathrm{TNG}$ telescope, which revealed the cluster nature, although deeper photometry would still be necessary for a final conclusion.

Isochrone fits are compatible with a nearly solar metallicity star cluster with age within the range 8-12 Gyr, which thus puts Pfleiderer 2 among the few metal-rich globular clusters in the Galaxy. Larger apertures would be important to get deeper imaging, as well as high-resolution spectroscopy to better derive its metallicity, abundance ratios, and orbital properties.

We thank the anonymous referee for suggestions. We acknowledge partial financial support from the Brazilian agencies CNPq and Fapesp, and Ministero dell'Università e della Ricerca Scientifica e Tecnologica (MURST), Italy.

\section{REFERENCES}

Andersen, M. I., Freyhammer, L., \& Storm, J. 1995, in Proc. ESO/ST-ECF Workshop on Calibrating and Understanding HST and ESO Instruments, ed P. Benvenuti (Garching: ESO), 87

Bica, E., \& Bonatto, C. 2008, MNRAS, 384, 1733

Bica, E., Bonatto, C., \& Camargo, D. 2008, MNRAS, 385, 349

Bonatto, C., \& Bica, E. 2007, MNRAS, 377, 1301

Dean, J. F., Warren, P. R., \& Cousins, A. W. J. 1978, MNRAS, 183, 569

Girardi, L., Bressan, A., Bertelli, G., \& Chiosi, C. 2000, A\&AS, 141, 371

Gnedin, O. Y., \& Ostriker, J. P. 1997, ApJ, 474, 223

Gratton, R. G., \& Ortolani, S. 1988, A\&AS, 73, 137

Harris, W. E. 1996, AJ, 112, 1487 (H96)

Innanen, K. A., Harris, W. E., \& Webbink, R. F. 1983, AJ, 88, 338

King, I. 1962, AJ, 67, 471

Kurtev, R., Ivanov, V. D., Borissova, J., \& Ortolani, S. 2008, A\&A, 489, 583

Landolt, A. U. 1983, AJ, 88, 439

Landolt, A. U. 1992, AJ, 104, 340

Lewis, M. S., Liu, W. M., Paust, N. E. Q., \& Chaboyer, B. 2006, AJ, 131, 2538 Ortolani, S., Barbuy, B., \& Bica, E. 1991, A\&A, 249, L31

Ortolani, S., Bica, E., \& Barbuy, B. 2006, ApJ, 646, L115

Ortolani, S., Rosino, L., \& Sandage, A. 1985, AJ, 90, 473

Pfleiderer, J., Weinberger, R., \& Mross, R. 1977, Star Cluster Symposium, Publ. Eotvos Univ., 5, 39

Reid, M. 1993, ARA\&A, 31, 345

Rosenberg, A., Saviane, I., Piotto, G., Aparicio, A., \& Zaggia, S. R. 1998, AJ 115,648

Salaris, M., \& Weiss, A. 2002, A\&A, 388, 492

Schlegel, D. J., Finkbeiner, D. P., \& Davis, M. 1998, ApJ, 500, 525

Stetson, P. B. 1987, PASP, 99, 191

Stetson, P. B. 1994, PASP, 106, 250

Strader, J., \& Kobulnicky, H. A. 2008, AJ, 136, 2120

Trager, S., King, I. R., \& Djorgovski, S. 1995, AJ, 109, 218 